\title{
Influence of Inhomogeneity of the Foundation on the Stress-strain State of the Design Located on it
}

\author{
Andrey Leontyev ${ }^{1, *}$, and Ksenia Balandina ${ }^{1}$ \\ ${ }^{1}$ Moscow State University of Civil Engineering, 26, Yaroslavskoye Shosse, Moscow, 129337, Russia
}

\begin{abstract}
The problem of the stress-strain state of the beam located on an inhomogeneous Winkler foundation is considered. It is accepted that the value of the coefficient of elasticity of the foundation has a symmetrical character relative to the middle of the beam and within the beam is represented by a quadratic law. The single-span beams with hinged supports along the edges are considered. For the calculation of beams on various types of symmetrically applied loads was used the program complex "Lira" and also Ritz-Timoshenko variational method. In the second case, the calculation was performed in Excel. Examples of calculation of short and long beams are considered. The effect of the degree of heterogeneity of the base on the deflections and bending moments of the beam under the action of concentrated and distributed loads is shown.
\end{abstract}

\section{Introduction}

The development of the theory of calculation of plates and beams located on an elastic Foundation can contribute to a more efficient and economical method of construction of all kinds of structures. The most common model describing the properties of the elastic base is the Winkler model [1]. Under this model, it is assumed that there is a direct proportional relationship between the load acting on the base $q_{0}(x)$ and his displacement $v(x)$ in the form: $q_{0}(x)=k_{0} \cdot v(x)$, where $k_{0}$ - coefficient of proportionality, termed "the coefficient of bed".

In the majority of works the basis is accepted uniform, however in practice it is often possible to observe change of size of coefficient of a bed on length of a design that influences the tensely-deformed condition of the beams located on such basis.

\section{State of the problem}

Let us assume that the value of the bed coefficient of the elastic foundation has a symmetrical change in relation to the middle of the beam, and within the beam is described

\footnotetext{
* Corresponding author: an leontiev@mail.ru
} 
by a quadratic law (figure 1). If the reference point is taken at the left end of the beam, the dependence of the coefficient of the bed from the coordinates will be written in the form:

$$
k(x)=\frac{\alpha}{10} \frac{12 k_{0}}{L^{2}}\left(x-\frac{L}{2}\right)^{2}+\frac{10-\alpha}{10} k_{0} .
$$

Here: $\alpha$ - the degree of heterogeneity of base, $L=2 l$ - the length of the beam,

$k_{0}$ - the average value of the coefficient of the bed, determined from the condition of equality of the areas of the graphs describing the resistance of the base, in the case of heterogeneous and homogeneous bases,

$k_{1}$ - the value of the coefficient of the bed at the left end of the beam: $k_{1}=k_{0}+k_{0} \cdot \alpha / 5$,

$k_{2}$ - the value of the coefficient of the bed under the center of the beam: $k_{2}=k_{0}(10-\alpha) / 10$.

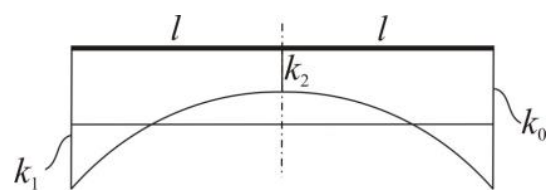

Fig. 1. Character of change of coefficient of the bed under a beam

The parameter $\alpha$ is introduced to indicate the degree of heterogeneity of the base, and in our case its value can vary from 0 to 10 (figure 2).

a)

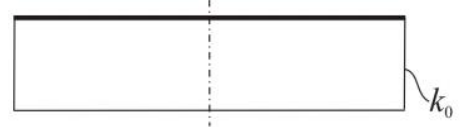

b)

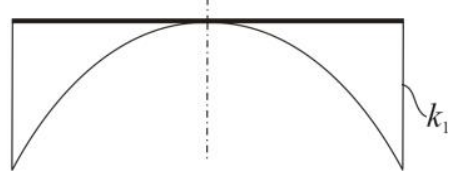

Fig. 2. Character of change of coefficient of the bed under a beam a) when $\alpha=0$, b) when $\alpha=10$

We consider single-span beams with hinged supports at the edges (figure 3 ).

a)

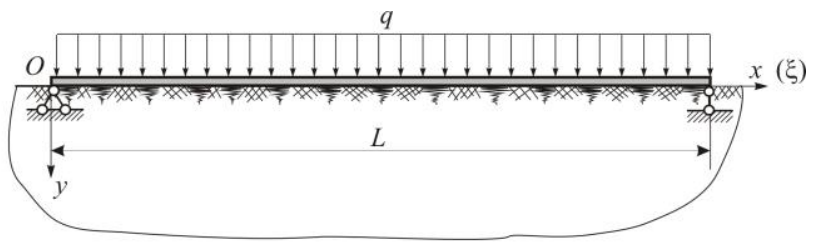

b)

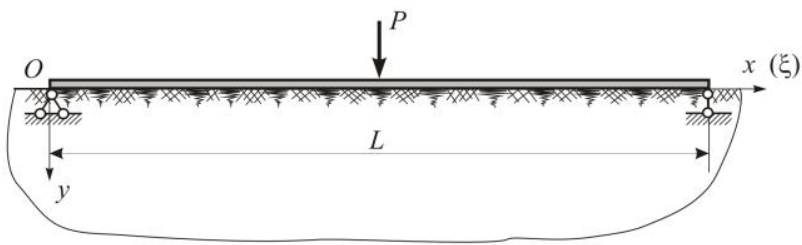

c)

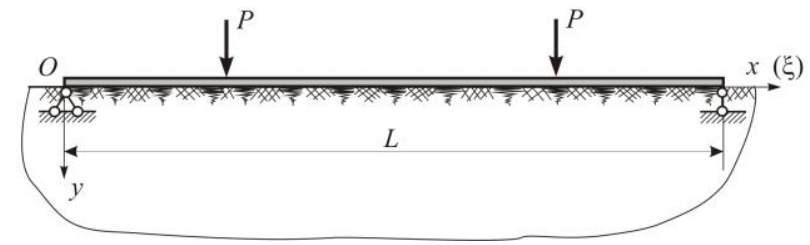

Fig. 3. Three types of symmetrical load 


\section{Analytical method of solving}

For calculation of the beams on different types of symmetrically applied loadings (figure 3) the variation method of Ritz-Timoshenko [2] was used.

For the general case of transverse bending of a beam on an elastic foundation, the potential energy is determined by the formula:

$$
U=\int_{0}^{L}\left[\frac{1}{2} \frac{M^{2}}{E J}+b k(x) \frac{v^{2}}{2}\right] d x-\int_{0}^{L} q v d x=\int_{0}^{L}\left[\frac{1}{2} \frac{\left(E J v^{I I}\right)^{2}}{E J}+b k(x) \frac{v^{2}}{2}\right] d x-\int_{0}^{L} q v d x .
$$

Here: $E$ - modulus of elasticity of beam material, $b$ - the width of the cross section of the beam, $J$ - his moment of inertia, $q$ - external load.

The deflection of the beam can be represented as a series:

$$
v(x)=a_{1} \varphi_{1}(x)+a_{2} \varphi_{2}(x)+\ldots .
$$

Substitute the series (3) in the expression of the energy (2) and write the condition of minimum potential energy (Lagrange):

$$
\frac{\partial U}{\partial a_{1}}=\int_{0}^{L}\left\{E J\left[a_{1}\left(\varphi_{1}\right)^{\prime \prime}+a_{2}\left(\varphi_{2}\right)^{\prime \prime}+\ldots\right]\left(\varphi_{1}\right)^{\prime \prime}+b k(x)\left(a_{1} \varphi_{1}+a_{2} \varphi_{2}+\ldots\right) \varphi_{1}\right\} d x-\int_{0}^{L} q \varphi_{1} d x=0 .
$$

In the case of orthogonality of the approximating functions for each number $n$ we obtain the equation of the form:

$$
a_{n} \int_{0}^{L}\left[E J\left(\varphi_{n}\right)^{\prime \prime}\left(\varphi_{n}\right)^{\prime \prime}+b k(x) \varphi_{n} \varphi_{n}\right] d x-\int_{0}^{L} q \varphi_{n} d x=0
$$

Sinusoids are taken as approximating functions: $\varphi_{n}(x)=\sin (n \pi x / L)$. This corresponds to the hinge conditions of the beam support.

Herewith

$$
\left(\varphi_{n}\right)^{\prime \prime}=-\left(\frac{n \pi}{L}\right)^{2} \sin \frac{n \pi x}{L}, \quad \quad \int_{0}^{L} \varphi_{n} \varphi_{n} d x=\frac{L}{2},
$$

and equation (5) takes the form:

$$
a_{n} \int_{0}^{L}\left[E J\left(\frac{n \pi}{L}\right)^{4}\left(\sin \frac{n \pi x}{L}\right)^{2}+b k(x)\left(\sin \frac{n \pi x}{L}\right)^{2}\right] d x-\int_{0}^{L} q \sin \frac{n \pi x}{L} d x=0 .
$$

We introduce the designation

$$
Q_{n}=\int_{0}^{L} q \sin \frac{n \pi x}{L} d x
$$

and substitute the expression (1) for $k(x)$ into the equation (6):

$$
a_{n} \int_{0}^{L}\left[E J\left(\frac{n \pi}{L}\right)^{4}\left(\sin \frac{n \pi x}{L}\right)^{2}+b\left[\frac{\alpha}{10} \frac{12 k_{0}}{L^{2}}\left(x-\frac{L}{2}\right)^{2}+\frac{10-\alpha}{10} k_{0}\right]\left(\sin \frac{n \pi x}{L}\right)^{2}\right] d x=Q_{n} .
$$

As a result of integration for series coefficients (3) we will have the following formula:

$$
a_{n}=\frac{2}{L} \frac{Q_{n}}{(n \pi)^{4}+4(\lambda L)^{4}\left(1-\frac{3}{5} \frac{\alpha}{n^{2} \pi^{2}}\right)} \frac{L^{4}}{E J} .
$$

\section{Examples of calculation}

Calculation by formulas (3) and (5) was performed in the Excel environment with a sufficiently large number of series members (3). Beams of different lengths were considered as examples. The influence of the base inhomogeneity degree on deflections and bending moments under the action of concentrated and distributed loads is analyzed. 
It is known that reference of a particular beam to one or another category (short, long) depends not on its actual length $L$, but on the product $\lambda L$, where the parameter $\lambda$ depends on beam stiffness and the coefficient of the bed: $\lambda=\sqrt[4]{\frac{k_{0} b}{4 E J}}$.

If $\lambda L<1,2$ the beam belongs to the category of absolutely rigid (stamp), if $1,2<\lambda L<2,2$ it can be considered short, if $2,2<\lambda L<3,7$ - medium beam, if $3,7<\lambda L<4,7$ - long beam.

In figure 4 the influence of the degree of heterogeneity of the base on the deflections of short beams, medium-length beams and long beams under the action of uniformly distributed load is shown (figure $3, \mathrm{a}$ ).

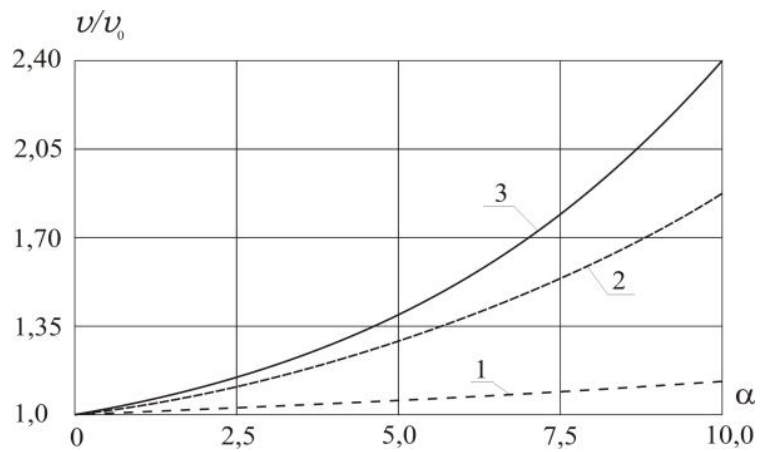

Fig. 4. The influence of the degree of heterogeneity of the base on the deflections in the center of the beam. 1. - short beam $(\lambda L=1.5), 2$. - beam of medium length $(\lambda L=3.0)$, 3. - long beam $(\lambda L=4.5)$

Here are the relative deflections in the center of the beam depending on the parameter $\alpha$, $v_{0}$ - deflection of the beam, located on a uniform foundation.

It can be seen that for short beams, the influence of the inhomogeneity of the base is not more than $13 \%$. For medium-length beams, this effect is already $70-90 \%$. For long beams, the deflection in the center of the beam on a non-uniform base can increase by 2 to 2.5 times compared to a homogeneous base.

Similar results were obtained for two other load types (figure 3,b,c), at the same time, the resultant of the external load remained constant.

As for the value of the bending moment in the center of the beam, the nature of the influence on it of the heterogeneity of the base depends on the type of load.

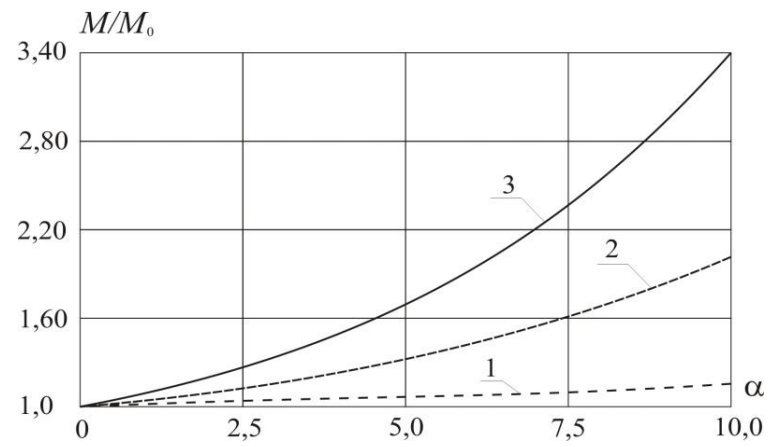

Fig. 5. The influence of the degree of heterogeneity of the base on the bending moment in the center of the beam. 1. - short beam $(\lambda L=1.5), 2$. - beam of medium length $(\lambda L=3.0), 3$. - long beam $(\lambda L=4.5)$ 
In figure 5 shows the influence of the degree of heterogeneity of the base on the value of the bending moment in the center of the beam for short beams, medium-length beams and long beams under uniformly distributed load (figure $3, \mathrm{a}$ ).

It can be seen that in this case, for short beams, the influence of the inhomogeneity of the base is not more than 14\%. For medium-length beams, the bending moment in the center of the beam can be increased by 2 times compared to the uniform base, and for long beams by $3-3.5$ times.

At action of the concentrated force applied in the center of a beam (figure 3,b), the influence of inhomogeneity of the base on the value of the bending moment in this section is significantly less than the uniformly distributed load (figure 6) and makes for short beams $10 \%$, for beams of average length $-60 \%$, for long beams $-80 \%$.

At action of two concentrated forces (figure 3,c) character of the plot of bending moments on length of a beam essentially depends on a way of application of loading that affects value of the bending moment in the center of the beam. For beams of different lengths, the calculation was performed using the program complex "Lira", the results of which are presented in figures 6-8. Here, taking into account the symmetry of the problem, the plots of bending moments for the right half of the beam are shown.

It can be seen that for short beams with maximum inhomogeneity $(\alpha=10)$ the bending moment in the center of the beam increases by $15 \%$ (figure 6 ).

a)

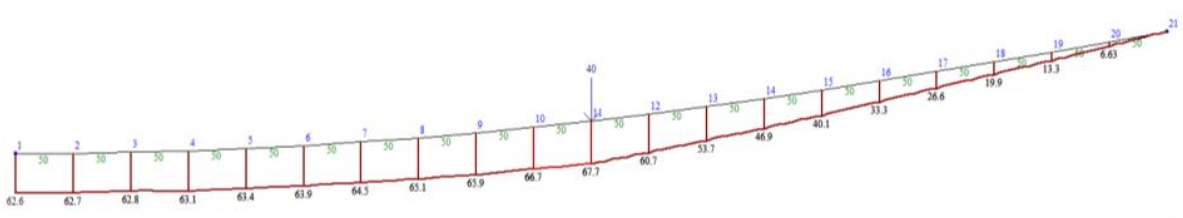

b)

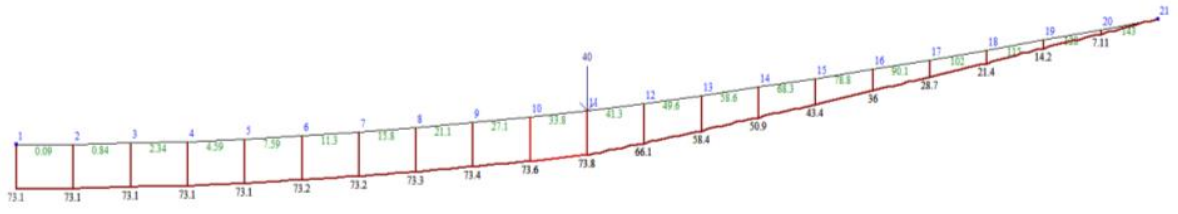

Fig. 6. Bending moment plots for short beam $(\lambda L=1.5)$ a) when $\alpha=0, b)$ when $\alpha=10$

For medium-length beams, the increase in bending moment occurs 3 times, mainly due to changes in the nature of the distribution of bending moments along the length of the beam (figure 7).

a)

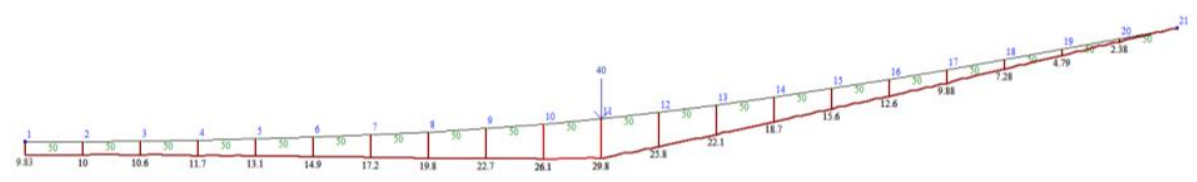

b

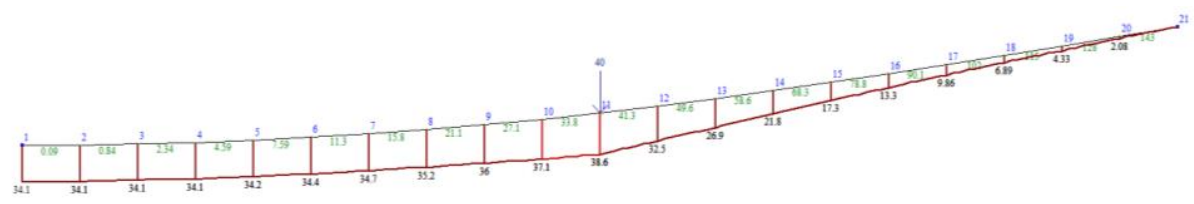

Fig. 7. Bending moment plots for beam of medium length $(\lambda L=3.0)$ a) when $\alpha=0$, b) when $\alpha=10$

For long beams, the distribution of bending moments along the beam length changes even more significantly (figure 8) 
a)

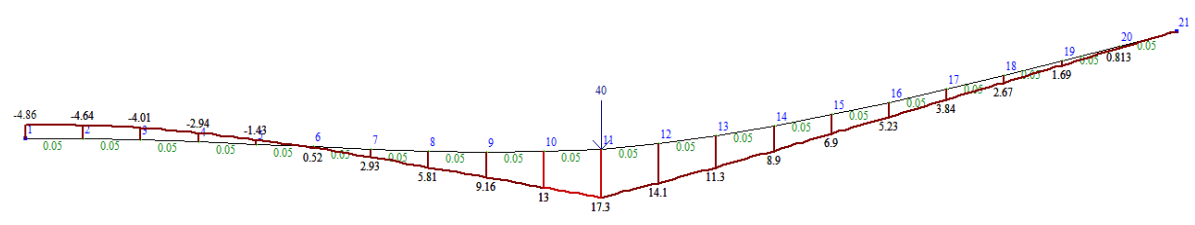

$\mathrm{b}$

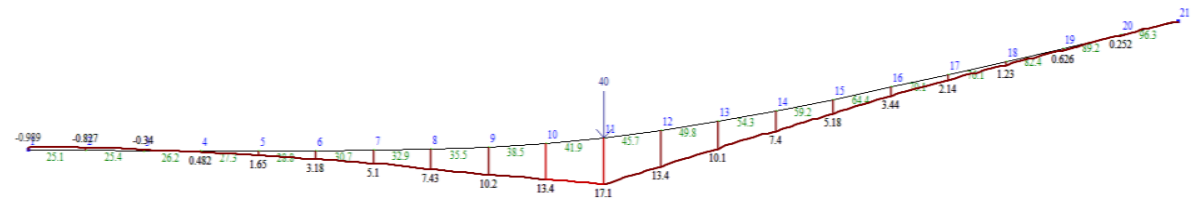

c)

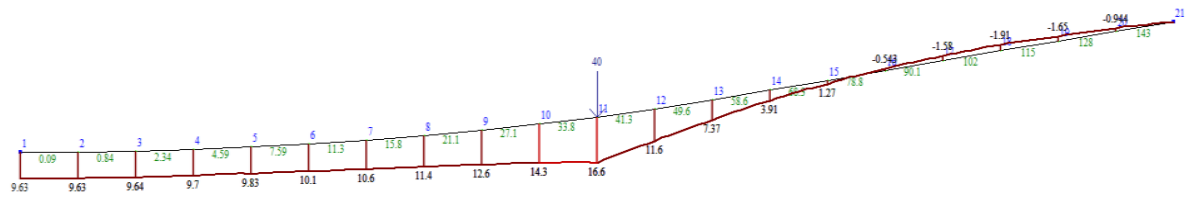

Fig. 8. Bending moment plots for long beam $(\lambda L=4.5)$ a) when $\alpha=0, b)$ when $\alpha=5$, c) when $\alpha=10$

\section{Conclusions}

The technique given in the article allows to take into account the influence of heterogeneity of the base on the stress-strain state of the structure located on it. The influence of rather essential inhomogeneity on the stress-strain state of beams of different length is shown on the examples.

It should be noted that the problems of calculation of structures on elastic foundation does not lose their relevance. The development of new models makes it possible to find engineering solutions that allow for analysis at the pre-project stage and to conduct a simpler analysis of the stress-strain state of structures and the ground base [4-7 etc.].

\section{References}

1. N.N. Leontiev and other, Fundamentals of the theory of beams and plates on a deformable base (MSUCE, 1982)

2. N.N. Leontiev, D.N. Sobolev, Variational principles of structural mechanics and basic theorems on elastic systems (MSUCE, 1980)

3. V.Z. Vlasov, N.N. Leontiev, Beams, plates and shells on elastic foundation (Fizmatlit, 1960)

4. V.I. Andreev, E.V. Barmenkova, A.V. Matveeva, Proc. of the Univ. Constr., 9 (2010)

5. V.I. Andreev, E.V. Barmenkova, Struct. Mech. and Calc. of Struct., 6 (2010)

6. V.I. Andreev, E.V. Barmenkova, Appl. Mech. and Mater., 204-208 (2012)

7. V.I. Andreev, A.V. Matveeva, E.V. Barmenkova, Appl. Mech. and Mater., 351-352 (2013) 